\title{
A logic of the similarity with prototypes and its relationship to fuzzy logic
}

\author{
Thomas Vetterlein
}

\author{
Johannes Kepler University Linz, Altenberger Straße 69, 4040 Linz, Austria
}

\begin{abstract}
Fuzzy sets are widely used to model vague properties. According to a common understanding, a fuzzy set represents the degrees of similarity of precisely specified objects with the prototypes of the considered vague property. We propose a logic based on this idea, using an entailment relation which was introduced in a work of Dubois, Prade, Esteva, Garcia, and Godo. The logic allows reasoning about the similarity with specific sets of prototypes; however, set-theoretic operations among the sets of prototypes associated to different properties are not dealt with. We arrive at a formalism closely related to fuzzy logic.
\end{abstract}

Keywords: Degree of similarity with a prototype, Logic of Approximate Entailment, interpretation of fuzzy logic.

\section{Introduction}

Let us assume that a fuzzy set is employed to model a vague property. We may for instance think of the property of humans to be "tall". Then, the domain of the fuzzy set refers to the aspect with respect to which we distinguish objects in order to tell whether they fulfil this property or not. To tell that somebody is "tall" means that the body height is taken into account; body heights may in turn be represented in centimetres by the positive reals [0, 250]. Furthermore, elements of the fuzzy set's domain which clearly fulfil the property in a given context are mapped to 1 and can be considered as prototypes for the property. In case of "tall", [180,250] could be chosen as the set of prototypes. The remaining elements are mapped to the degree to which they are compatible with the property under consideration. This degree may be viewed as the similarity of an element with the set of prototypes. E.g., in a model of "tall", 178 is not much different from 180 and could be assigned the degree 0.8 .

This is a well-known interpretation of fuzzy sets; "similarity" is the key notion. For the similaritybased interpretation of fuzzy sets as well as other possible interpretations, we refer, e.g., to $[5,6]$. The idea to understand fuzzy sets as crisp sets together with a similarity relation is furthermore developed in [7]. The approach has also been found particular useful in the field of pattern classification; the presumably earliest contribution in this direction is [2]. The idea is in fact widely applicable - namely whenever vagueness plays a role.

The notion of similarity is dealt with in the framework of approximate reasoning, which goes back to Ruspini's basic paper [14]. Ruspini's ideas have been elaborated in a number of papers from a logical perspective $[7,8,9,10]$. Here, we take up an approach proposed in [7]. The formalism is closely related to the Logic of Approximate Entailment, which was proposed in Rodriguez's Thesis [13].

The idea of the latter logic is to formalise implicational relationships in an approximate way. The framework is a similarity space $(W, s)$, where $W$ is a set of worlds and $s$ is a similarity relation on $W$; propositions are interpreted as subsets of $W$ and graded implications

$$
\alpha \stackrel{c}{\Rightarrow} \beta
$$

are considered, where $c$ is a real number between 0 and 1. $\alpha$ and $\beta$ being interpreted by $A, B \subseteq W$, respectively, (1) is satisfied if

$$
A \subseteq U_{c}(B),
$$

where $U_{c}(B)$ is the $c$-neighbourhood of $B$, containing all worlds similar to $B$ at least to the degree $c$. Thus, for $\alpha \stackrel{c}{\Rightarrow} \beta$ to be satisfied, the set $A$ need not necessarily be contained in $B$, but in a set which may be considered as similar to $B$ to the degree $c$.

In this contribution, we modify the Logic of Approximate Entailment, aiming at a means to reason in the presence of vagueness. We exploit the idea that a vague property can be modelled by the set of its prototypes together with a relation expressing the similarity with the prototypes.

\section{The setting}

Let us explain in an informal way our procedure and ideas. For further material and explanations, consult, e.g., [7, 10].

We start as usual with a set of worlds $W$, representing the set of possible circumstances which we are going to consider and reason about. The properties characterising worlds are assumed to be in general vague. With each property $\alpha$, we associate a pair $(A, s)$ consisting of a set $A \subseteq W$ and a fuzzy relation $s: W \times W \rightarrow[0,1]$. $A$ represents the set of prototypes of $\alpha ; s$ measures the degree of similarity of two worlds with respect to the aspect of distinction associated with $\alpha$. 
For example, consider the above mentioned case that $\alpha$ models "tall". Our set of worlds is then $S=[0,250]$, representing the set of heights of humans, and $A=[180,250]$. The role of $s$ is to tell to which extent a difference in height matters for the applicability of "tall"; if $x, y \in W$ represent two heights and $x$ is assigned "tall", then $y$ should be compatible with the property "tall" at least to the degree $s(x, y)$. A possibility is to define $s(x, y)=\max \left\{1-\frac{|x-y|}{10}, 0\right\}$; then $s$ is a similarity relation w.r.t. the Łukasiewicz t-norm.

We can then tell to which degree a precise height $x$ fits to the notion "tall"; namely, we let this degree be the similarity of $x$ with $A$, or more precisely, with the point in $A$ which is closest to $x$ w.r.t. $s$. We arrive in this way at a fuzzy set $u$, as it could well be used to model "tall":

$$
u: S \rightarrow[0,1], \quad x \mapsto \begin{cases}0 & \text { if } x \leq 170, \\ \frac{x-170}{10} & \text { if } 170 \leq x \leq 180, \\ 1 & \text { if } x \geq 180 .\end{cases}
$$

In our setting, however, we do not in general require $s$ to be a similarity relation. A similarity relation maps a pair of worlds to 1 exactly in case that the two worlds coincide. This is acceptable if we include a single aspect of distinction, like the height of a certain person, into consideration. But the discourse may address more than one aspect. For instance, the height of a second person may be taken into account for comparative purposes. The set of worlds would then be $S \times S$, with the above definition of $S$, and two worlds may differ with respect to the height of one but not the other person. In order to reason about both persons' heights it is reasonable to take into account two fuzzy relations, each of which refers to one of the persons. But then, the separability condition is to be dropped.

Consequently, we will model each variable $\alpha$ by a pair $(A, s)$ consisting of a set $A$ of worlds and a pseudosimilarity relation $s$, where the prefix "pseudo" expresses the fact that separability is not assumed. In particular, each variable in our language will be assigned an own pseudosimilarity relation. The tnorm $\odot$ appearing in the transitivity condition will however be one and the same for all relations. In fact, we will assume here that $\odot$ is the Eukasiewicz t-norm; then each pseudosimilarity relation will be the dual of a bounded pseudometric.

Let us now describe the type of statements which we are going to deal with. We will, first of all, consider implicational relationships: we will formalise statements of the form that the Boolean combination of certain properties implies another such combination. The meaning of these implications is to be understood in terms of the prototypes of the addressed properties. As however the modelled properties are assumed to be vague, tolerance will be incorporated into the formalism. We do so in a double way. On the one hand, we assign each implication an explicit degree, expressing that the im- plication holds only in a rough way. On the other hand, we require stability of the implication under small changes. In both cases the pseudosimilarities which are part of the interpretation of the language are used.

For instance, let $\alpha$ and $\beta$ be variables. The implication $\alpha \stackrel{c}{\Rightarrow} \beta$ means informally that $\alpha$ implies $\beta$. This implication is subject to coarse-grainedness; it holds the more loosely the smaller the value $c$ is. On the formal side, let $\alpha$ and $\beta$ be interpreted by $(A, s)$ and $(B, t)$, respectively. Tolerance in the relationship between $\alpha$ and $\beta$ will be realised, on the one hand, in the same way as in the Logic of Approximate Entailment. If $\alpha \stackrel{c}{\Rightarrow} \beta$ are satisfied by the indicated interpretation, then

$$
A \subseteq U_{c}^{t}(B)
$$

where $U_{c}^{t}(B)$ is the $c$-neighbourhood of $B$ with respect to the pseudosimilarity $t$.

On the other hand, the implication is subject to a continuity condition. In accordance with the vagueness of the property denoted by $\alpha$, a change of $A$ should not possibly invalidate the implication between $\alpha$ and $\beta$; the implication is required to hold under a change of $B$ to the same extent. Our actual definition of satisfaction is consequently stronger than (2) and is given as follows:

$$
U_{d}^{s}(A) \subseteq U_{c \odot d}^{t}(B) \quad \text { for all } d .
$$

Condition (3) coincides with what is called $d$ proximity entailment in [7]. (2) says that a world in which $\alpha$ holds is compatible with $\beta$ to the degree $\geq c$. (3) moreover says that a world which is compatible with $\alpha$ to the degree $\geq d$ is compatible with $\beta$ to the degree $\geq c \odot d$. Clearly, (3) includes (2) as a special case.

In the particular case $c=1$ we have $A \subseteq B$. However, we have more: in addition $U_{d}^{s}(A) \subseteq U_{d}^{t}(B)$ holds also for non-one degrees $d$. In contrast, $\alpha \stackrel{0}{\Rightarrow} \beta$ always holds, that is, implications to the degree 0 do not express anything.

The interpretation (3) is in line with [7, Def. 2]. We note, moreover, that there are connections to applications other than approximate reasoning. The paper [5] proposes ways to model comparative rules of the form "the more $\alpha$ is the case, the more $\beta$ is the case." Our formalism presents a possibility among those proposed in [5].

What makes our procedure special from the point of view of approximate reasoning, but leads to wellknown ideas from the point of view of fuzzy logic, is the treatment of compound formulas. In this respect, we do not follow the lines of [7] and related papers. In fact, we do not consider Boolean combinations of the crisp sets representing prototypes of properties.

The variables of our language refer to possibly vague properties and we assume that it is exactly these properties with respect to which it is possible 
to evaluate a degree of similarity. Consider the case of a medical expert system; assume that its knowledge base consists of if-then rules of the form that the presence of certain symptoms implies the presence of a certain disease. For example, a rule may say that the symptoms $\sigma_{1}$ and $\sigma_{2}$ imply the disease $\delta$. In our framework, this rule will be expressible by the implication

$$
\sigma_{1} \wedge \sigma_{2} \stackrel{1}{\Rightarrow} \delta
$$

Let now the symptoms be interpreted by $\left(S_{1}, s_{1}\right)$ and $\left(S_{2}, s_{2}\right)$, respectively. An actual world $w$ be given, a natural way to use (4) would be to determine how similar $w$ is with the intersection of the sets of prototypes associated to $\sigma_{1}$ and $\sigma_{2}$, that is, how similar $w$ is with $S_{1} \cap S_{2}$. The similarity relation used would in some way be constructed from $s_{1}$ and $s_{2}$.

Here, we understand (4) differently. We do not work with set-theoretic combinations of the sets of prototypes, but only with classical logical combinations of statements about similarities. Namely, we require that if, for some $t \in[0,1]$, the actual world $w$ is w.r.t. $s_{1}$ similar with $S_{1}$ to the degree $t$ and w.r.t. $s_{2}$ similar with $S_{2}$ to the degree $t$, then $w$ is similar to the degree $t$ with the set of prototypes associated with $\delta$. The emphasized "and" corresponds to the " $\wedge$ " connective in (4).

In particular, the interpretation (3) applies for atomic formulas only. Implications involving compound formulas are ordinary Boolean combinations of statements involving the degrees of similarity with the crisp sets assigned to atomic formulas.

More technically speaking, we proceed as follows. Assume that variables $\alpha$ and $\beta$ are interpreted by $(A, s)$ and $(B, t)$, respectively. We thereby associate with $\alpha$ and $\beta$ a system of neighbourhoods around the set of prototypes, with respect to a similarity associated individually to $\alpha$ and $\beta$. To interpret, e.g., $\alpha$, we use $U_{c}^{s}(A)$, where $c$ runs over the real unit interval. For a fixed $c, U_{c}^{s}(A)$ contains all worlds which are with respect to the aspect of distinction associated to $\alpha$ compatible with $\alpha$ to a degree at least $c$.

To the compound proposition $\alpha \wedge \beta$, we equally associate a system of nested sets. Simply, for each $c$, this set contains those worlds which are compatible with both $\alpha$ and $\beta$ to the degree at least $c$. So we take the set of worlds which are compatible with $\alpha$ to a degree $\geq c$ and also with $\beta$ to a degree $\geq c$, where the degree refers to the respective pseudosimilarity: $U_{c}^{s}(A) \cap U_{c}^{t}(B)$. Similarly, to the proposition $\alpha \vee \beta$, we associate, for each $c$, those worlds which are compatible either with $\alpha$ or $\beta$ to a degree $\geq c$ : we take $U_{c}^{s}(A) \cup U_{c}^{t}(B)$. Finally, consider the proposition $\sim \alpha$. We want to associate to $\sim \alpha$ the worlds $w$ such that $w$ fits to non- $\alpha$ to the degree at least $c$. To this end, we take those worlds which fit to $\alpha$ at most to the degree $1-c$.
As a result, for example, the formula

$$
\alpha \wedge \sim \beta \stackrel{c}{\Rightarrow} \gamma \vee \delta
$$

has the following interpretation. If, for any $d$, our actual world $w$ is similar to (a prototype of the property denoted by) $\alpha$ at least to the degree $d$ and similar to $\beta$ to a degree at most $1-d$, then $w$ is similar to the degree $c \odot d$ either to $\gamma$ or to $\delta$.

We would like to point out that our logic is not designed to force artifically a similarity with fuzzy logic. Consider the above example from medicine and assume that $\sigma_{1}$ and $\sigma_{2}$ denote independently observed symptoms, like "high fever" and "stiffness of the neck". Then we may say that a patient has high fever to a certain degree, and a stiff neck to a certain degree; and we may interpret both these degrees as similarities and draw our conclusions from the two values. It is however questionable to say that the patient's state is similar to a certain degree to the conjunction of "high fever" and "stiffness of the neck" and to require to draw our conclusion from a single value; after all, we are concerned with two different means of observations. Two different observation cannot in general be combined, that is, expressed on the basis of a single notion, for the sake of their further procession.

\section{The logic AF}

Based on the informal considerations and decisions of the preceding section, we proceed as follows.

$\odot$ always denotes the Eukasiewicz t-norm.

Definition 3.1. Let $W$ be a non-empty set. Then $s: W \times W \rightarrow[0,1]$ is a pseudosimilarity if for all $x, y, z \in W$ (i) $s(x, x)=1$, (ii) $s(x, y)=s(y, x)$, and (iii) $s(x, z) \geq s(x, y) \odot s(y, z)$. We denote by $\hat{1}$ the constant 1 pseudosimilarity.

For $x \in W$ and $A \subseteq W$, we define the similarity of $x$ with $A$ by

$$
\delta^{s}(x, A)=\sup \{s(x, y): y \in A\},
$$

and we define the $c$-neighbourhood of $A$ w.r.t. $s$ by

$$
U_{c}^{s}(A)=\left\{x \in W: \delta^{s}(x, A) \geq c\right\}
$$

for any $c \in[0,1]$.

We define the Logic AF (where "A" refers to approximate entailment and " $F$ " refers to fuzzy logic) semantically as follows. The language will be finite; $N \geq 1$ is fixed.

Definition 3.2. The propositions of AF are built up from variables $\varphi_{1}, \ldots, \varphi_{N}$ and the constants $\perp, \top$ by means of the binary connectives $\wedge, \vee$ and the unary connective $\neg$. An implication of AF is a triple consisting of a finite set $\alpha_{1}, \ldots, \alpha_{n}, n \geq 1$, a single proposition $\beta$, and a real value $c \in[0,1]$, written

$$
\alpha_{1}, \ldots, \alpha_{n} \stackrel{c}{\Rightarrow} \beta
$$


$c$ is called the tolerance value.

Let $W$ be a non-empty set. An evaluation for AF on $W$ assigns to each variable a pair $(A, s)$ consisting of a non-empty subset $A$ of $W$ and a pseudosimilarity $s$ on $W$, and to the constants $\perp, \top$ the pairs $(\emptyset, \hat{1}),(W, \hat{1})$, respectively.

Let $v$ be an evaluation on a set $W$. For any $c \in[0,1]$, the mapping $\mathcal{U}_{c}$ from the set of propositions to the power set of $W$ is defined recursively as follows: if $\alpha$ is an atom, $\mathcal{U}_{c}(\alpha)=U_{c}^{s}(A)$, where $v(\alpha)=(A, s)$; for any propositions $\alpha$ and $\beta$, $\mathcal{U}_{c}(\alpha \wedge \beta)=\mathcal{U}_{c}(\alpha) \cap \mathcal{U}_{c}(\beta), \mathcal{U}_{c}(\alpha \vee \beta)=\mathcal{U}_{c}(\alpha) \cup \mathcal{U}_{c}(\beta)$, and $\mathcal{U}_{c}(\neg \alpha)=W \backslash \bigcup_{d>1-c} \mathcal{U}_{d}(\alpha)$.

An implication $\alpha_{1}, \ldots, \alpha_{n} \stackrel{c}{\Rightarrow} \beta$ is satisfied by $v$ if $\mathcal{U}_{d}\left(\alpha_{1}\right) \cap \ldots \cap \mathcal{U}_{d}\left(\alpha_{n}\right) \subseteq \mathcal{U}_{c \odot d}(\beta)$ for all $d \in[0,1]$

A theory $\mathcal{T}$ of AF is a finite set of implications of $\mathrm{AF}$, and $\mathcal{T}$ is said to semantically entail a further implication $\varphi$ of AF if $\varphi$ is satisfied by any evaluation satisfying all elements of $\mathcal{T}$.

We are going to establish the "fuzzy-logical character" of AF. An unrestricted identification of AF with a fuzzy logic seems not to be possible. When saying so, we assume that fuzzy logic, according to the guidelines contained in the fundamental monograph [11], deals with logics whose semantics can be based on the real unit interval. However, if we restrict to tautologies, there is a direct connection between AF and a particular fuzzy logic, which we will define next.

We will use the symbols $\wedge, \vee, \sim$ to denote the minimum, maximum, and standard negation on the real unit interval, respectively.

Definition 3.3. Propositions and implications of $\mathrm{AF}^{\prime}$ are defined like for $\mathrm{AF}$. An $\mathrm{AF}^{\prime}$-evaluation is a mapping $w$ from the set of propositions to the real unit interval such that $w(\alpha \wedge \beta)=w(\alpha) \wedge w(\beta)$, $w(\alpha \vee \beta)=w(\alpha) \vee w(\beta), w(\neg \alpha)=\sim w(\alpha), w(\perp)=$ $0, w(\top)=1$.

An $\mathrm{AF}^{\prime}$-evaluation $w$ satisfies an implication $\alpha_{1}, \ldots, \alpha_{n} \stackrel{c}{\Rightarrow} \beta$ if $\left(w\left(\alpha_{n}\right) \wedge \ldots \wedge w\left(\alpha_{n}\right)\right) \odot s \leq w(\beta)$.

A theory $\mathcal{T}$ is said to $\mathrm{AF}^{\prime}$-semantically entail an implication $\varphi$ of AF if $\varphi$ is satisfied by any $\mathrm{AF}^{\prime}$ evaluation satisfying all members of $\mathcal{T}$.

For an evaluation to satisfy a theory, we mean that each element of the theory is satisfied. For a theory to possess a model, we mean that there is at least one evaluation satisfying $\mathcal{T}$.

Theorem 3.4. Let $\mathcal{T}$ be a theory of AF such that $\mathcal{T}$ possesses at least one model. Then $\mathcal{T}$ semantically entails an implication $\varphi$ if and only if $\mathcal{T} \mathrm{AF}^{\prime}$ semantically entails $\varphi$.

Proof. Assume first that $\mathcal{T}$ does not semantically entail $\varphi$. Let $v$ be an evaluation on $W$ satisfying $\mathcal{T}$ but not $\varphi$. To each variable $\alpha$, interpreted by the pair $(A, s)$, we associate the fuzzy set

$$
f(\alpha): W \rightarrow[0,1], \quad x \mapsto \delta^{s}(x, A) .
$$

Moreover, we extend $f$ to all propositions such that $f(\alpha \wedge \beta)=f(\alpha) \wedge f(\beta), f(\alpha \vee \beta)=f(\alpha) \vee f(\beta)$, and $f(\neg \alpha)=\sim f(\alpha)$. Here, the pointwise minimum, maximum, standard negation of fuzzy sets if denoted by $\wedge, \vee, \neg$, respectively.

We claim that then $\mathcal{U}_{c}(\alpha)=[f(\alpha)]^{c}, c \in[0,1]$. Indeed, this is the case for atoms by construction. For the general case, we argue by induction. Assume that $\mathcal{U}_{c}(\alpha)=[f(\alpha)]^{c}$ and $\mathcal{U}_{c}(\beta)=[f(\beta)]^{c}$ holds for arbitrary propositions $\alpha, \beta$ and all $c \in[0,1]$. Then

$$
\begin{aligned}
\mathcal{U}_{c}(\alpha \wedge \beta)= & \mathcal{U}_{c}(\alpha) \cap \mathcal{U}_{c}(\beta) \\
= & {[f(\alpha)]^{c} \cap[f(\beta)]^{c} } \\
= & \{x \in W: f(\alpha)(x) \geq c\} \\
& \cap\{x \in W: f(\beta)(x) \geq c\} \\
= & \{x:(f(\alpha) \wedge f(\beta))(x) \geq c\} \\
= & \{x: f(\alpha \wedge \beta)(x) \geq c\} \\
= & {[f(\alpha \wedge \beta)]^{c} . }
\end{aligned}
$$

Similarly, we argue for the $\vee$ connective. Finally, for any $x \in W$, we have

$$
\begin{aligned}
x \in \mathcal{U}_{c}(\neg \alpha) & \\
& \text { iff } \neg(\exists d>1-c)\left(x \in \mathcal{U}_{d}(\alpha)\right) \\
& \text { iff } \neg(\exists d>1-c)(f(\alpha)(x) \geq d) \\
& \text { iff }(\forall d>1-c)(f(\alpha)(x)<d) \\
& \text { iff } f(\alpha)(x) \leq 1-c \\
& \text { iff } \sim f(\alpha)(x) \geq c \\
& \text { iff } x \in[\sim f(\alpha)]^{c} \\
& \text { iff } x \in[f(\neg \alpha)]^{c} .
\end{aligned}
$$

Hence an implication $\alpha \stackrel{c}{\Rightarrow} \beta$ is satisfied by $v$ iff $\mathcal{U}_{d}(\alpha) \subseteq \mathcal{U}_{d \odot c}(\beta)$ for all $d \in[0,1]$ iff $[f(\alpha)]^{d} \subseteq$ $[f(\beta)]^{d \odot c}$ for all $d \in[0,1]$ iff $f(\alpha)(x) \geq d$ implies $f(\beta)(x) \geq d \odot c$ for all $d \in[0,1]$ and $x \in W$ iff $f(\alpha)(x) \odot c \leq f(\beta)(x)$ for all $x \in W$. Moreover, $f(\cdot)(x)$ is an $\mathrm{AF}^{\prime}$-evaluation for any $x \in W$. This fact applied to $\mathcal{T}$ and $\varphi$, we see that there is an $\mathrm{AF}^{\prime}$-evaluation satisfying $\mathcal{T}$ but not $\varphi$.

Assume now that $\mathcal{T}$ does semantically entail $\varphi$. Let furthermore $\bar{w}$ be an $\mathrm{AF}^{\prime}$-evaluation satisfying $\mathcal{T}$. We have to show that $\bar{w}$ satisfies $\varphi$.

We shall construct an evaluation $v$ satisfying $\mathcal{T}$ as follows. Let $W$ be the set of all $\mathrm{AF}^{\prime}$-evaluations satisfying $\mathcal{T}$; then $W$ can be considered as a subset of $[0,1]^{N}$, where $N$ is the number of variables. To a variable $\alpha$, we assign the set $A$ of those AF' $^{\prime}$ evaluations $w$ such that $w(\alpha)=1$, and the relation $s$ defined by $s\left(w_{1}, w_{2}\right)=1-\left|w_{1}(\alpha)-w_{2}(\alpha)\right|$ for $w_{1}, w_{2} \in W$. Clearly, $s$ is a pseudosimilarity on $W$. We claim that $A$ is non-empty. Indeed, as $W$ is a closed subset of $[0,1]^{N}$, there would otherwise be a $d<1$ such that $w(\alpha) \leq d$ for all $w$ satisfying $\mathcal{T}$. This means that $\mathcal{T}$ would $A F^{\prime}$-semantically entail $\alpha \stackrel{1-d}{\Rightarrow} \perp$, and by the first part of this theorem, $\mathcal{T}$ would semantically entail $\alpha \stackrel{1-d}{\Rightarrow} \perp$. But $1-d>0$ implies that there is no evaluation for AF satisfying 
$\alpha \stackrel{1-d}{\Rightarrow} \perp$. This is a contradiction to the assumption that $\mathcal{T}$ possesses at least one model.

Thus $v$ is an evaluation. Define $f$ as above; then, as we have seen above, an implication $\alpha \stackrel{c}{\Rightarrow} \beta$ is satisfied by $v$ iff $f(\alpha)(w) \odot c \leq f(\beta)(w)$ for all $w \in$ $W$. Moreover, $f(\alpha)(w)=w(\alpha)$ for any variable $\alpha$ and consequently, by an induction argument, for all propositions $\alpha$. It follows that an implication is satisfied by $v$ iff satisfied by each $w \in W$. In particular, $v$ satisfies $\mathcal{T}$. By assumption, $v$ then also satisfies $\varphi$. In particular, $\varphi$ is satisfied by $\bar{w} \in$ $W$.

Theorem 3.4 suggests that the logics AF and $\mathrm{AF}^{\prime}$ actually coincide. This is however not the case; note that Theorem 3.4 depends on the assumption that the considered theory possesses a model of AF. Indeed, the coincidence is prevented by the fact that in AF, each variable must be interpreted by a fuzzy set with a non-empty kernel.

However, the situation is different with regard to tautologies, as we will see next.

\section{A proof system for the AF-tautologies}

From Theorem 3.4, we observe that tautologies of $\mathrm{AF}$ and $\mathrm{AF}^{\prime}$ coincide.

Theorem 4.1. An implication $\varphi$ is satisfied by any evaluation of $\mathrm{AF}$ if and only if $\varphi$ is satisfied by any $\mathrm{AF}^{\prime}$-evaluation.

Proof. This follows from Theorem 3.4 given the fact that the empty theory always possesses a model.

On the basis of Theorem 4.1 it is possible to provide an analytic proof system for AF, with which we can derive the tautologies.

The procedure is, all in all, routine. Our proof system makes use of hypersequents; see, e.g., [12]. In fact, we use, in particular for the completenes proof below, techniques similar to the case of Gödel logic or to the logic GZL, which both resemble $\mathrm{AF}^{\prime}$ in some respects and were studied in [1] and [3], respectively.

Hypersequents are multisets of sequents; sequents in turn coincide in our case syntactically with implications of AF.

Definition 4.2. The rules of AF are the following, where $\alpha, \beta$ are propositions, $\Gamma$ is a finite set of propositions, $c, d \in[0,1]$, and $\mathcal{G}$ is a hypersequent:

Axioms

$$
\begin{aligned}
& \overline{\perp \stackrel{c}{\Rightarrow} \alpha}(A 1) \quad \overline{\alpha \stackrel{c}{\Rightarrow} \alpha}(A 2) \\
& \overline{\alpha \stackrel{c}{\Rightarrow} \top}(A 3) \quad \overline{\alpha \stackrel{0}{\Rightarrow} \beta}(A 4)
\end{aligned}
$$

Logical rules

$$
\frac{\mathcal{G} \mid \Gamma, \alpha, \beta \stackrel{c}{\Rightarrow} \gamma}{\mathcal{G} \mid \Gamma, \alpha \wedge \beta \stackrel{c}{\Rightarrow} \gamma}(\wedge \rightarrow) \quad \frac{\mathcal{G} \mid \Gamma \stackrel{c}{\Rightarrow} \gamma}{\mathcal{G} \mid \Gamma, \alpha \stackrel{c}{\Rightarrow} \gamma}(\mathrm{lw})
$$

$$
\frac{\mathcal{G}|\Gamma \stackrel{c}{\Rightarrow} \alpha \quad \mathcal{G}| \Gamma \stackrel{c}{\Rightarrow} \beta}{\mathcal{G} \mid \Gamma \stackrel{c}{\Rightarrow} \alpha \wedge \beta}(\rightarrow \wedge)
$$

$$
\begin{aligned}
& \frac{\mathcal{G}|\Gamma, \alpha \stackrel{c}{\Rightarrow} \gamma \quad \mathcal{G}| \Gamma, \beta \stackrel{c}{\Rightarrow} \gamma}{\mathcal{G} \mid \Gamma, \alpha \vee \beta \stackrel{c}{\Rightarrow} \gamma}(\vee \rightarrow) \\
& \frac{\mathcal{G} \mid \Gamma \stackrel{c}{\Rightarrow} \alpha}{\mathcal{G} \mid \Gamma \stackrel{c}{\Rightarrow} \alpha \vee \beta}(\rightarrow \vee)
\end{aligned}
$$

$$
\begin{gathered}
\frac{\mathcal{G} \mid \neg \beta \stackrel{c}{\Rightarrow} \alpha}{\mathcal{G} \mid \neg \alpha \stackrel{c}{\Rightarrow} \beta}(\neg \rightarrow) \quad \frac{\mathcal{G} \mid \beta \stackrel{c}{\Rightarrow} \neg \alpha}{\mathcal{G} \mid \alpha \stackrel{c}{\Rightarrow} \neg \beta}(\rightarrow \neg) \\
\frac{\mathcal{G} \mid \beta \stackrel{c}{\Rightarrow} \alpha}{\mathcal{G} \mid \neg \alpha \stackrel{c}{\Rightarrow} \neg \beta}(\neg \rightarrow \neg)
\end{gathered}
$$

Rules for the tolerance value

$$
\begin{gathered}
\frac{\mathcal{G} \mid \Gamma \stackrel{c}{\Rightarrow} \alpha}{\mathcal{G} \mid \Gamma \stackrel{d}{\Rightarrow} \alpha}(\mathrm{c}), \text { where } d \leq c \\
\frac{\mathcal{G} \mid \alpha \stackrel{\frac{c}{2}}{\Rightarrow} \perp}{\mathcal{G} \mid \alpha \stackrel{c}{\Rightarrow} \neg \alpha}\left(\rightarrow \frac{1}{2}\right) \quad \frac{\mathcal{G} \mid \top \stackrel{\frac{c}{2}}{\Rightarrow} \alpha}{\mathcal{G} \mid \neg \alpha \stackrel{c}{\Rightarrow} \alpha}\left(\frac{1}{2} \rightarrow\right)
\end{gathered}
$$

Structural rules

$$
\begin{gathered}
\frac{\mathcal{G}|\Gamma \stackrel{c}{\Rightarrow} \alpha| \Gamma \stackrel{c}{\Rightarrow} \alpha}{\mathcal{G} \mid \Gamma \stackrel{c}{\Rightarrow} \alpha}(\mathrm{EC}) \\
\frac{\mathcal{G}}{\mathcal{G} \mid \Gamma \stackrel{c}{\Rightarrow} \alpha}(\mathrm{EW}) \\
\frac{\mathcal{G}\left|\alpha \stackrel{c_{1}}{\Rightarrow} \beta \quad \mathcal{G}\right| \gamma \stackrel{c_{2}}{\Rightarrow} \delta}{\mathcal{G}\left|\gamma \stackrel{d_{1}}{\Rightarrow} \beta\right| \alpha \stackrel{d_{2}}{\Rightarrow} \delta}(\mathrm{Com}) \\
\frac{\mathcal{G}\left|\Gamma_{1} \stackrel{c}{\Rightarrow} \alpha \quad \underset{\mathcal{G}}{\Rightarrow}\right| \Gamma_{2}, \alpha \stackrel{d}{\Rightarrow} \beta}{\mathcal{G} \mid \Gamma_{1}, \Gamma_{2} \stackrel{c \bigodot}{\Rightarrow} \beta} \text { (Trans) }
\end{gathered}
$$

The definition of a proof in AF is as usual.

Note that the peculiar (Com) rule has the consequence that the following rule is derivable in AF:

$$
\frac{\mathcal{G} \mid \alpha \stackrel{c \odot d}{\Rightarrow} \beta}{\mathcal{G}|\alpha \stackrel{c}{\Rightarrow} \gamma| \gamma \stackrel{d}{\Rightarrow} \beta}
$$

Furthermore, if we use the (Trans) rule for the case that the cut formula is a constant, we arrive at the following rules:

$$
\frac{\mathrm{T} \stackrel{c}{\Rightarrow} \alpha}{\beta \stackrel{c}{\Rightarrow} \alpha}(\mathrm{R} 2) \quad \frac{\alpha \stackrel{c}{\Rightarrow} \perp}{\alpha \stackrel{c}{\Rightarrow} \beta}
$$

In what follows, an implication of AF will be called valid if satisfied by any evaluation.

Moreover, a literal is a proposition of the form $\alpha$ or $\sim \alpha$, where $\alpha$ is atomic. 
Theorem 4.3. An implication $\varphi$ of $\mathrm{AF}$ is valid if and only if $\varphi$ is provable in $\mathrm{AF}$. We can moreover assume that (Trans) is replaced by the rules (R2) and (R3).

Proof. By Theorem 4.1, we may prove the Theorem with reference to $\mathrm{AF}^{\prime}$ rather than $\mathrm{AF}$.

The soundness of the rules with respect to $\mathrm{AF}^{\prime}$ evaluations is not difficult to check.

Assume that we are given an implication $\varphi$ which is valid in $A F^{\prime}$, i.e., satisfied by all $A F^{\prime}$-evaluations. There are derivable rules for $\wedge$ and $\vee$ which are invertible; we may apply them backwards and get valid hypersequents in which no binary connective and no comma appears.

Let us consider one such hypersequent $\mathcal{H}$. If in some sequent of $\mathcal{H}$ the same literal appears on both sides, $\mathcal{H}$ is derivable by (A2) and (EW). If else in some sequent the same variable appears on both sides, we can achieve, by backwards application of the $\frac{1}{2}$-rules, that this variable appears only on one side of the sequent.

If no variable is present, $\mathcal{H}$ is derivable by (A1), (A3), or (A4). If $\mathcal{H}$ contains at least one variable, we show that we may construct from $\mathcal{H}$ another hypersequent $\mathcal{H}^{\prime}$ from which $\mathcal{H}$ is derivable and which contains one variable less. The claim then follows by induction.

Assume that $\mathcal{H}$ contains the variable $\alpha$. We can achieve, by backwards application of the $\neg$-rules, that this variable appears only non-negated. If $\alpha$ appears always on the left side or always on the right side, we replace $\alpha$ by $\top$ or $\perp$, respectively; in view of (R2) and (R3), we are done.

Assume now that $\alpha$ appears at least once on the left side and at least once on the right side. We discuss the case that $\alpha$ appears twice on the left side and twice on the right side; the general case is analogous. Thus we assume that $\mathcal{H}$ is of the form

$$
\mathcal{G}\left|\beta_{1} \stackrel{d_{1}}{\Rightarrow} \alpha\right| \beta_{2} \stackrel{d_{2}}{\Rightarrow} \alpha\left|\alpha \stackrel{e_{1}}{\Rightarrow} \gamma_{1}\right| \alpha \stackrel{e_{2}}{\Rightarrow} \gamma_{2}
$$

for some literals $\beta_{1}, \beta_{2}, \gamma_{1}, \gamma_{2}$ and some $\mathcal{G}$ in which $\alpha$ does not appear. Any $\mathrm{AF}^{\prime}$-evaluation $w$ satisfies $\mathcal{G}$ or fulfils the condition

$$
\begin{array}{r}
w\left(\beta_{1}\right)-\sim d_{1} \leq w(\alpha) \\
\text { or } w\left(\beta_{2}\right)-\sim d_{2} \leq w(\alpha) \\
\text { or } w(\alpha) \leq w\left(\gamma_{1}\right)+\sim e_{1} \\
\text { or } w(\alpha) \leq w\left(\gamma_{2}\right)+\sim e_{2} .
\end{array}
$$

Consequently, any $\mathrm{AF}^{\prime}$-evaluation $w$ satisfies $\mathcal{G}$ or fulfils

$$
\begin{aligned}
& \left(w\left(\beta_{1}\right)-\sim d_{1}\right) \wedge\left(w\left(\beta_{2}\right)-\sim d_{2}\right) \\
\leq & \left(w\left(\gamma_{1}\right)+\sim e_{1}\right) \vee\left(w\left(\gamma_{2}\right)+\sim e_{2}\right) ;
\end{aligned}
$$

indeed, otherwise some $\mathrm{AF}^{\prime}$-evaluation $w$ would not satisfy $\mathcal{G}$ and would fulfil $\left(w\left(\gamma_{1}\right)+\sim e_{1}\right) \vee\left(w\left(\gamma_{2}\right)+\right.$ $\left.\sim e_{2}\right)<\left(w\left(\beta_{1}\right)-\sim d_{1}\right) \wedge\left(w\left(\beta_{2}\right)-\sim d_{2}\right)$; hence, adjusting the value $w(\alpha)$ if necessary, there would be an $\mathrm{AF}^{\prime}$-evaluation not satisfying $\mathcal{G}$ and not fulfilling (5).

Thus any $w$ satisfies $\mathcal{G}$ or fulfils

$$
\begin{aligned}
& w\left(\beta_{1}\right)-\sim d_{1} \leq w\left(\gamma_{1}\right)+\sim e_{1} \\
& \text { or } w\left(\beta_{1}\right)-\sim d_{1} \leq w\left(\gamma_{2}\right)+\sim e_{2} \\
& \text { or } w\left(\beta_{2}\right)-\sim d_{2} \leq w\left(\gamma_{1}\right)+\sim e_{1} \\
& \text { or } w\left(\beta_{2}\right)-\sim d_{2} \leq w\left(\gamma_{2}\right)+\sim e_{2},
\end{aligned}
$$

that is,

$$
\begin{aligned}
w\left(\beta_{1}\right) \odot d_{1} \odot e_{1} & \leq w\left(\gamma_{1}\right) \\
\text { or } w\left(\beta_{1}\right) \odot d_{1} \odot e_{2} & \leq w\left(\gamma_{2}\right) \\
\text { or } w\left(\beta_{2}\right) \odot d_{2} \odot e_{1} & \leq w\left(\gamma_{1}\right) \\
\text { or } w\left(\beta_{2}\right) \odot d_{2} \odot e_{2} & \leq w\left(\gamma_{2}\right) .
\end{aligned}
$$

We conclude that the hypersequent $\mathcal{H}^{\prime}$

$$
\begin{gathered}
\mathcal{G}\left|\beta_{1} \stackrel{d_{1} \odot e_{1}}{\rightrightarrows} \gamma_{1}\right| \beta_{1} \stackrel{d_{1} \odot e_{2}}{\Rightarrow} \gamma_{2} \mid \\
\beta_{2} \stackrel{d_{2} \odot e_{1}}{\Rightarrow} \gamma_{1} \mid \beta_{2} \stackrel{d_{2} \odot e_{2}}{\Rightarrow} \gamma_{2}
\end{gathered}
$$

is valid as well. Furthermore, we may derive $\mathcal{H}$ from $\mathcal{H}^{\prime}$ by (R1) and (EC).

\section{Conclusion}

We have proposed a particular way to deal formally with vague information. The proposed method leads directly to the realm of mathematical fuzzy logic. Our proposal is however accompanied by a particular justification.

We have argued as follows. Vague properties are commonly modelled by fuzzy sets. The fuzzy set used for this purpose may arise as follows. First of all, it possesses a non-empty kernel; the kernel represents the prototypical cases. Moreover, the elements mapping to values strictly between 0 and 1 are located, intuitively speaking, around the kernel; they represent the borderline cases. The remaining elements represent the cases of total incompatibility with the modelled property. Thus it is reasonable understand a fuzzy set as its kernel together with a similarity relation such that the similarity of an element with the kernel equals the value to which it is mapped by the fuzzy set.

This understanding of fuzzy sets is common; see, e.g., $[4,7]$. Our impression is however that the idea has not yet been much explored in the framework of formal logic. As we have shown, the interpretation of fuzzy sets in the indicated sense leads, first of all, to a practically usable propositional calculus. Several choices need certainly to be made. But if done in the way proposed here, we arrive at the calculus $\mathrm{AF}$ which, in a restricted sense, can be called a fuzzy logic - even if we prescribe a fuzzy logic to be based on linearly ordered semantics.

The logic AF provides a bridge between fuzzy logic and the Logic of Approximate Entailment [13]. Indeed, in view of its intended semantics AF is conceptually closely related to this variant of Ruspini's 
formalism for approximate reasoning. However, in the way how compound formulas are treated, AF comes close to fuzzy logic.

For AF, we have proved a weak completeness theorem. To prove strong completeness remains as a task for further work. Note that the proof system presented here is not ideal. An interpretation of the hypersequents such that the rules presented above are sound might be possible; it would amount, however, to a distinction of worlds by cases. Consequently, a proof would in general hardly be interpretable in an informal manner. A redefined proof system for AF should not be based on hypersequents. Effort into this direction are certainly not in line with the common practice in fuzzy logic - again in case that we understand fuzzy logics as logics associated necessarily with linearly ordered structures.

Otherwise, one might also consider to modify or generalize our setting. When modelling a statement like (4), there are three values which have to be related to each other: the degree to which the actual facts resemble to the prototypes of the three properties referred to by the three variables. Depending on the application, it might make sense to consider other relationships than the one which we have chosen. In this case, the whole universe of ideas appearing in mathematical fuzzy logic could be applied. For instance, the conjunction could be chosen in a way differently from the minimum and $\odot$ could be interpreted in a way different from Eukasiewicz tnorm.

\section{References}

[1] M. Baaz, C. G. Fermüller, Analytic Calculi for Projective Logics, in: N. V. Murray (ed.), Automated reasoning with analytic tableaux and related methods, Proceedings of TABLEAUX '99, Springer-Verlag, Berlin 1999; pages 36-50.

[2] R. Bellman, L. Kalaba, L. A. Zadeh, Abstraction and pattern classification, J. Math. Anal. Appl. 13:1-7, 1966.

[3] A. Ciabattoni, T. Vetterlein, On the (fuzzy) logical content of CADIAG-2, Fuzzy Sets Syst. 161:1941-1958, 2010.

[4] D. Dubois, Gradual inference rules in approximate reasoning, Inf. Sci. 61:103-122, 1992.

[5] D. Dubois, H. Prade, The three semantics of fuzzy sets, Fuzzy Sets Syst. 90:141-150, 1997.

[6] D. Dubois, H. Prade, Similarity versus preference in fuzzy set-based logics, in: E. Orłowska (ed.), Incomplete Information: Rough Set Analysis, Physica-Verlag, Heidelberg 1998; pages 441-461.

[7] D. Dubois, H. Prade, F. Esteva, P. Garcia, L. Godo, A logical approach to interpolation based on similarity relations, Int. J. Approx. Reasoning 17:1-36, 1997.
[8] F. Esteva, P. Garcia, L. Godo, R. O. Rodríguez, A modal account of similarity-based reasoning, Int. J. Approx. Reasoning 16:235260, 1997.

[9] F. Esteva, L. Godo, R. O. Rodríguez, T. Vetterlein, Logics for approximate and strong entailment, submitted.

[10] L. Godo, R. O. Rodríguez, Logical approaches to fuzzy similarity-based reasoning: an overview, in: G. Della Riccia et al., editors, Preferences and similarities, Springer-Verlag, Berlin 2008, pages 75-128.

[11] P. Hájek, Metamathematics of Fuzzy Logic, Kluwer Acad. Publ., Dordrecht 1998.

[12] G. Metcalfe, N. Olivetti, D. Gabbay, Proof theory for fuzzy logics, Springer-Verlag, Dordrecht 2009.

[13] R. O. Rodríguez, Aspectos formales en el Razonamiento basado en Relaciones de Similitud Borrosas, Ph.D. Thesis, Technical University of Catalonia (UPC), 2002.

[14] E. Ruspini, On the semantics of fuzzy logic, Int. J. Approx. Reasoning 5:45-88, 1991. 